\title{
Tilted Pulsar Beams
}

\section{Geoff Wright and Patrick Weltevrede}

Jodrell Bank Centre for Astrophysics, Alan Turing Building, University of Manchester, Manchester, M13 9PL

\begin{abstract}
In 1975 the carousel model was proposed by Ruderman \& Sutherland to explain the beautiful phenomenon of drifting subpulses. However the simultaneous appearance of subpulse bands which drift in opposing directions - a feature now found in two pulsars - is difficult to reconcile with this model, both geometrically and physically. Here we propose a geometric resolution of this problem which also may shed light on a range of previously baffling phenomena. The model places significant constraints on the underlying physics of pulsar emission.
\end{abstract}

Keywords. pulsars: general, pulsars: individual (PSR J0815+09,PSR B1839-04)

\section{Introduction}

Many pulsars have emission patterns which are thought to arise when our sightline passes through a beam formed by one or two carousels of sub-beams, although problems remain in understanding how in many cases the carousels apparently suddenly accelerate and change the observed pattern. However, the phenomenon of "bi-drifting" found in two pulsars with very typical pulsar parameters is not just a physical problem: it would seem to contradict the basic geometric idea of a circular carousel. This problem is discussed in detail by Weltevrede (2016).

\section{The model}

It is proposed that the beams of many, if not all, pulsars take an elliptical form which is tilted to a greater or lesser extent with respect to the fiducial plane. Bi-drifting is then observed in special cases when the circulating sub-beams are traversed by a sightline which is close to the meridional plane (low impact angle $\beta$ ). The fact that such pulsars are rare suggests that for most pulsars the beam is near-circular and/or is only weakly tilted.

Here we show simulations (Fig. 1) of the two known examples of bi-drifting. In both cases two nested carousels are assumed, precisely elliptical in form with the same number of sub-beams in each. We cannot be sure that the chosen parameters are optimal or unique representations of the data, only that they are consistent with the data (Wright \& Weltevrede 2017). Our point is to demonstrate that a good approximation to the observations can be achieved without abandoning the idea of a closed carousel.

Since it is generally assumed that the precise sightline traverse across a pulsar beam depends on frequency, one prediction of the model is that bi-drifting may be observed at higher/lower frequency but not at the lower/higher frequency.

\section{Wider Implications}

Asymmetric profiles. Elliptical structures on the polar cap combined with a symmetric radius-to-frequency mapping will generate asymmetric and frequency-dependent pulsar profiles, a feature found in many pulsars. 

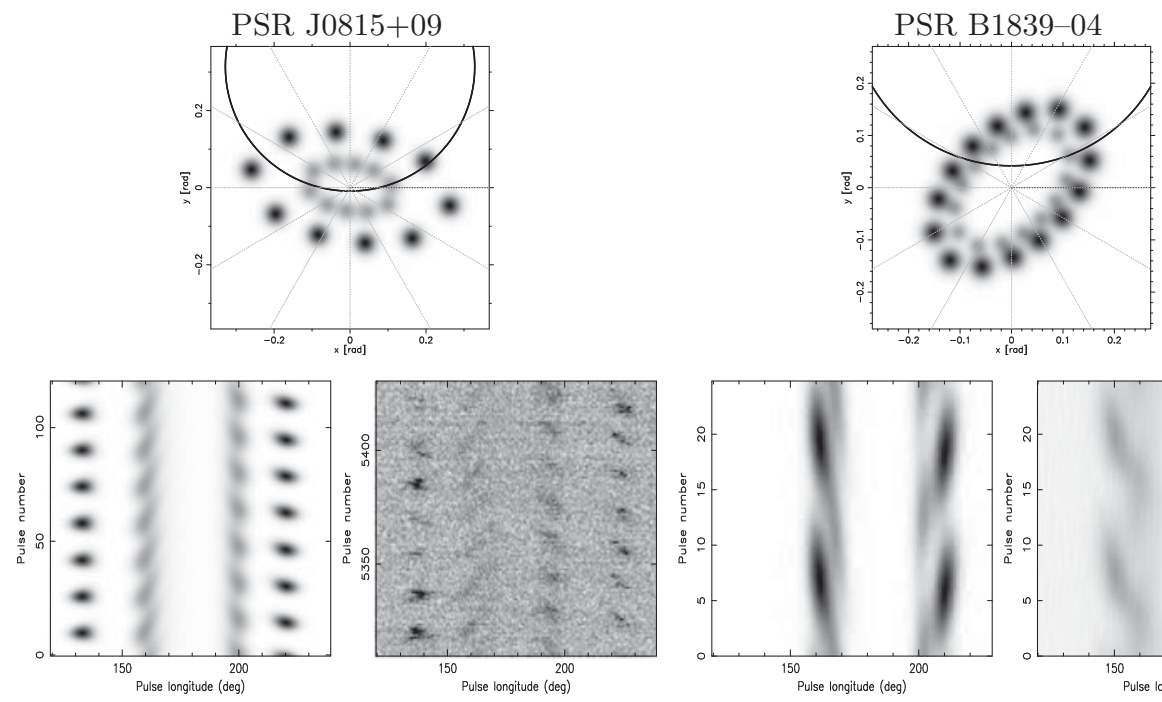

Figure 1. A comparison of the simulation with the observations of PSRs J0815+09 (left) and B1839-04 (right). top: The titled elliptical beams and the line of sight indicated by the solid line. bottom: Emission drifts in opposing senses in different components (for each pulsar, the simulation is left, the observed pulses right). Figure from Wright \& Weltevrede 2017.

"Flare" pulsars. These pulsars shift their emission to earlier longitudes on irregular timescales (e.g. Perera et al. 2015). We suggest that these may be due to sudden or gradual changes in the tilt of the oval beam.

Mode-changing. Many pulsars are known to switch within a few pulses from one mode of behaviour to another, accompanied by a sudden change in profile (e.g. Bilous et al. 2014). This may be due to a change in the alignment of the carousel.

\section{Conclusions and Physical Interpretations}

By abandoning the rigid assumption that pulsars have circular beams we have been able to show that a wide range of phenomena from bi-drifting to asymmetric profiles and even moding and flare stars can be given a geometric framework.

At first sight the resolution of the bi-drift phenomenon as a carousel of non-circular form may seem to give support to the classical polar cap model. However, it is not easy to see why "sparks" on a polar cap in the presence of magnetic multipole components would arrange themselves in such a way that they form closed matching nested loops.

Alternatively, some feedback with the magnetosphere is at work, a feature which is already supported by the known inter-pole coordination seen in a number of highlyinclined pulsars.

\section{References}

Bilous, A. V. et al., 2014, A\&A A, 572, 52

Champion,D. J., et al. 2005, ApJ, 363, 929

Perera, B. B. P.., et al. 2016, MNRAS, 446, 1380

Ruderman, M.. \& Sutherland, P. 1975, ApJ, 196, 51

Weltevrede, P. 2016, A\& A, 464, 2597

Wright, G. \& Weltevrede, P. 2017, MNRAS, 464, 2597 